\title{
Exploration and practice of teaching reform on photoelectric comprehensive experiments
}

Wei Wang, Bing Lei, Jianhua Shi, Wei Liu

Wei Wang, Bing Lei, Jianhua Shi, Wei Liu, "Exploration and practice of teaching reform on photoelectric comprehensive experiments," Proc. SPIE 11143, Fifteenth Conference on Education and Training in Optics and

Photonics: ETOP 2019, 111433G (2 July 2019); doi: 10.1117/12.2523738

SPIE Event: Fifteenth Conference on Education and Training in Optics and Photonics: ETOP 2019, 2019, Quebec City, Quebec, Canada 


\title{
Exploration and practice of teaching in photoelectric comprehensive experiments
}

\author{
Wei Wang*, Bing Lei, Jianhua Shi, Wei Liu \\ College of Advanced Interdisciplinary Studies, National University of Defense Technology, \\ Changsha, China
}

\begin{abstract}
With the high-speed development of science and technology, students in colleges and universities are required to improve their practical and innovative abilities, especially for those majored in photoelectric engineering. However, it is difficult to achieve this goal if we only employ theoretical teaching and traditional experiments through which students are only required to verify some theorems and conclusions through the established operations. To address this issue, comprehensive experiments are adopted in many colleges and universities to enhance the students' ability of knowledge application and independent innovation. In this paper, an experiment, in which students are asked to design a system to detect the rotational speed, rotational direction and stop bit of a flywheel in real time by optoelectronic measurement, is given as an example to introduce the teaching reform of comprehensive experiments from three aspects including experimental design, teaching method and experimental evaluation. In experimental design, compared with traditional comprehensive experiments, we pay more attention to the practicability in project and the comprehensiveness of knowledge. For an experiment, its practical value can arouse students' interest, and it should cover as much professional knowledge as possible to improve the students' ability of synthetical knowledge application. In the course of experiment, to meet the requirement of cultivating students' self-learning ability, the experimental teaching method is adjusted from teacher-centered into student-centered. Besides, a scientific and reasonable experimental evaluation system is needed to motivate students to do experiments positively. Therefore, a new multifaceted evaluation system is set up to test students' grasp of theoretical knowledge, knowledge application ability, practical ability, innovation ability and team working ability comprehensively. Through five years of exploration and practice accumulation, our teaching mode of comprehensive experiments is verified to be effective to develop students' capability of knowledge application, practice and innovation.
\end{abstract}

Keywords: photoelectric comprehensive experiments, practical ability, innovation ability, quality education

\section{INTRODUCTION}

The experimental teaching is an important part in the Higher Education which aims to cultivate students with a wide range of abilities encompassing cognitive, practical and innovation abilities ${ }^{1}$. However, the traditional experiments are usually the verification, that is, students only need to verify some theory through existing experimental apparatus. Apparently, they can't meet the requirements of talent training because their content is lack of stimulation and challenge to the development of students' intelligence ${ }^{2,3}$. To this end, a number of colleges and universities set up a new type of experiments named comprehensive experiments as an essential part of practical teaching. The purpose of comprehensive experiments is to provide an open experimental condition and environment to allow students to design a system by their own. It is much better to develop the ability of students than through the verifying experiment. In this way, students' indepth understanding of knowledge, knowledge application ability, practical ability, troubleshooting and problem solving skills, and innovation ability can be greatly improved. Despite comprehensive experiments have been set up for several years in many colleges and universities, such as Zhejiang University, China University of Mining and Technology, University of Electronic Science and Technology of China and so on, there are still some problems existing in the comprehensive teaching as follows:

1. Practical is not well. An important purpose of experiments is to encourage students to put the knowledge what they have learned into practice, and a comprehensive experiment with a strong practical significance can enhance students' enthusiasm to use their knowledge to solve real problems. However, it often be neglected by the designers of comprehensive experiments.

Fifteenth Conference on Education and Training in Optics and Photonics: ETOP 2019, edited by

Anne-Sophie Poulin-Girard, Joseph A. Shaw, Proc. of SPIE Vol. 11143, 111433G · ( 2019

SPIE, ICO, IEEE, OSA·CCC code: 0277-786X/19/\$18 · doi: 10.1117/12.2523738 
2. Lack of comprehensiveness. The comprehensive experiment, which is treated as the promotion and expansion of professional foundation courses, should be a synthesis of basic theory knowledge of several courses. In other words, it should cover a wide range of knowledge. Unfortunately, the comprehensiveness is rarely considered while many comprehensive experiments are designed. Consequently, they can't efficiently promote students' ability of synthetical knowledge application.

3. Limited by traditional teaching mode. The aim of comprehensive experiments is to help teachers to cultivate students' ability of learning on their own and solving the practical problems autonomously, but sometimes the goal can't be achieved as the tradition teaching mode are still adopt by some teachers. In the course, students are given too detailed instructions and rely too much on teachers if they have troubles in their experiments, therefore, their all-round ability can't be improved.

4. Imperfect experimental evaluation. A scientific and reasonable experimental evaluation is not only an important mean to judge teaching effect, but it is also an effective way to correct students' attitude towards experiments. Despite the traditional experimental evaluation system, in which the final results of students are mainly determined by experimental data and reports, is simple and can be easily operated, it is difficult to mobilize the enthusiasm of students.

Confronted with the problems described above, it is urgent for us to take reform in the comprehensive experimental teaching to strength the students' practical and innovation ability. In this paper, a new comprehensive experimental teaching mode is introduced while a photoelectric comprehensive experiment is taken as an example.

\section{REFORM OF COMPREHENSIVE EXPERIMENTAL TEACHING}

In this section, a photoelectric comprehensive experiment is given as an example to introduce a new mode of comprehensive experiment teaching from three aspects: experimental design, teaching method and experimental evaluation.

\subsection{Experimental designing with practicability in project and comprehensiveness of knowledge}

The practicability in project and comprehensiveness of knowledge should be taken into account when we design a comprehensive experiment. The practical value of the experiment plays an important role to stimulate students' interest. In addition, for the purpose of improving students' ability of synthetical knowledge application, a good comprehensive experiment should involve knowledge of several professional courses.

According to the above principles, a new photoelectric comprehensive experiment is designed. Rotational speed and direction measurement is widely used in the field of aviation, machine, precision machinery manufacturing, and so on ${ }^{4}$. Therefore, in the experiment, students are required to build a photoelectric detection system to detect the rotational speed, rotational direction and stop bit of a flywheel in real time. Rotational speed measurement is widely used in engineering. For example, we need to measure the speed of motor, alternator, spin axis of machine tools in real-time when they are rotating. Compared with mechanical measurements which have low accuracy, complex system and poor stability, the measurement based on photoelectric detection is steady, reliable, better in real-time and higher in accuracy. Generally, in the experiment, the speed, direction and stop bit are measured based on the electric signals transformed from the optical signals which are reflected by the reflective stripes on the flywheel. The experimental scheme is given in Figure 1. The light reflected by the reflective stripes on the flywheel is received and transformed into electric by a photoelectric detector, then the electric signal is amplified and reshaped into a square wave, based on which a single chip finally complete the measurement task and display the results in real-time. Obviously, this comprehensive experiment involves knowledge of light sources, photoelectric detectors, analog circuits, programs and so on. It provides a good opportunity for students to construct their comprehensive knowledge system. 


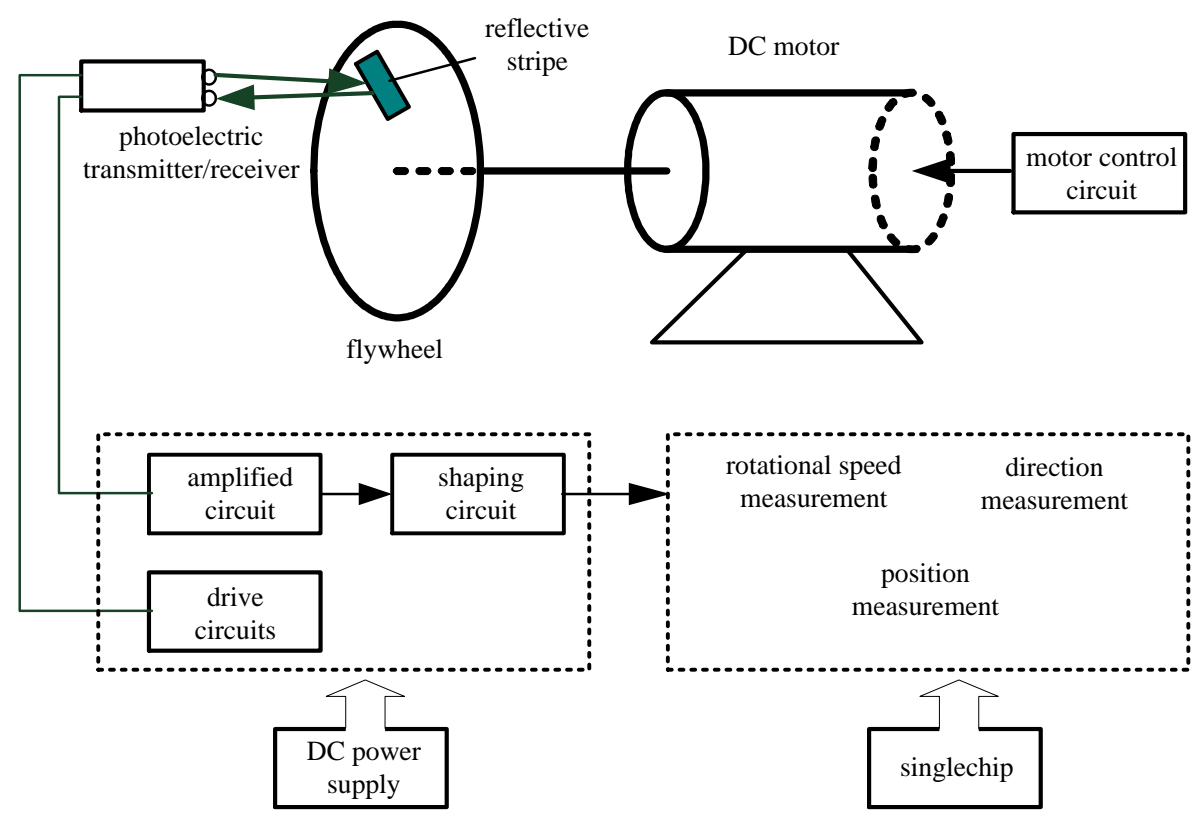

Figure 1. The illustration of one possible experimental scheme.

\subsection{Student-centered experimental teaching method}

Studies have shown that teacher-centered teaching may be the easiest teaching method, but it is not the best learning method. By traditional teacher-centered teaching, teachers introduce the experimental principle and method as detailed as possible, and students are expected to follow teachers' instruction to do the experiment. In this case, though all students can complete the experiment, their practical ability can't be improved and innovative thought can't be stimulated. Comparing to the traditional teacher-centered teaching, student-centered teaching is a modern teaching method which treats students as the main body of teaching ${ }^{5}$. In our student-centered experimental teaching, we adopt the following methods:

1. Students are encouraged to complete the experiment independently. Before the experiment is conducted, teachers only introduce the practical value, task and scheme of the experiment. Specially, the scheme should be described briefly. Teachers only provide the structure figure of the system (as shown in Figure 1) and explain the function of each module in it. However, the realization of these functions is not explained. Students should solve problems by their own. For example, we only explain why an amplifier and reshaping module is needed, but we don't give a design of the module. Students should deal with it using their analog circuit knowledge. Besides, we emphasize to students that the scheme shown in Fig.

2. Teachers should use heuristic teaching method to guide students to think and create in the process of experiment. When students ask for help when they have troubles with their experiments, teachers should give heuristic guidance rather than detailed solution. For instance, students may ask teachers to help them when there are no square waves output from a 555 chip. In fact, the reason is that the maximum of the voltage signal input to the chip is less than $10 / 3 \mathrm{~V}$ according to the lack of an amplifier circuit. Instead of pointing out the problem directly, we ask students some questions such as, do you really understand the reshaping principle of a 555 chip, is there any requirement on the input signal, how to meet the requirement, and so on. Following these questions, students can get the answers by their own. In addition, during this process, the students' ability of solving problem is highly improved. 1 is only one possible solution, and they can design other schemes.

3. Various experiment equipment, electronic elements and experimental material are provided to avoid limiting students' creativity. In practice, there are a variety of solutions to a problem. For example, light sources and photoelectric detectors have their own spectral characteristics, to make sure the photoelectric detector can detect the light reflected by the reflective strips, one important factor is the compatibility of the spectral characteristics of light sources and photoelectric detectors. If the students are only given one kind of light sources and photoelectric detectors, they will use it without considering why this kind of light source and photoelectric detector can work well and what are the requirements for 
choosing them. For this reason, in our experiment teaching, several kinds of light sources and photoelectric detectors are provided along with their spectrum characters. Students need to choose the matched light sources and photoelectric detectors depending on the spectrum characters of the devices. Along this way, their understanding and application of spectrum characters of light sources and photoelectric detectors are highly enhanced. Furthermore, despite that the light sources and photoelectric detectors with compatible spectrum characters all can be used, the light source emitting infrared light with the wavelength at $890 \mathrm{~nm}$ and its matched photoelectric detector (i.e., the detector which has high photoelectric transformation efficiency at $890 \mathrm{~nm}$ ) have the optimal performance. The result is that, the interference of background light can be eliminated while infrared light is chosen. In the experiment, students are encouraged to choose the devices with the best performance according to analysis in theory or experiment and investigate to discover the reason. This process is of much help in cultivating students' ability of independent study and analysis of problem.

\subsection{Process emphasized experimental evaluation}

The experimental evaluation is used to evaluate students' learning effects, what is more, it can be used to cultivate students into generalists ${ }^{6}$. For this purpose, a scientific and reasonable experimental evaluation is proposed to evaluate students' ability and performance completely. The result of evaluation consists of scheme, operation, performance, report and presentation (as shown in Figure 2).

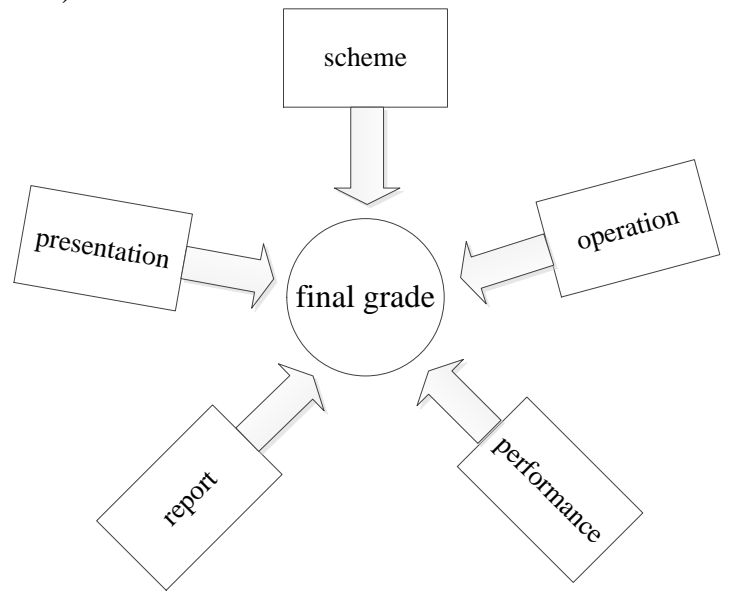

Figure 2. The construction of final grade.

To improve students' innovative ability, the evaluation on scheme which account for $15 \%$ of the final grade pays attention to the feasibility and innovation of the scheme. At the beginning of the class, a scheme is introduced to students without the detailed design of each module, students can get an underlying score if they choose the scheme and complete the detailed design. Students who design a new feasible scheme can get a higher score. Table 1 gives the scoring rule.

Table 1. The scoring rule of scheme.

\begin{tabular}{|c|c|}
\hline rule & score (total score is 15) \\
\hline validity & $0 \sim 4$ \\
\hline care & $0 \sim 3$ \\
\hline innovation & $0 \sim 8$ \\
\hline
\end{tabular}

To attach great importance to operation which reflects students' ability of practice, the evaluation on operation takes up $40 \%$ of the final grade. The evaluation on operation takes independence, the ability of analyzing and solving problems and team spirit into consider. The scoring rule is given in Table 2.

Table 2. The scoring rule of operation.

\begin{tabular}{|c|c|}
\hline rule & score (total score is 40) \\
\hline independence & $0 \sim 7$ \\
\hline normalization & $0 \sim 7$ \\
\hline the ability of analyzing and solving problems & $0 \sim 16$ \\
\hline team spirit & $0 \sim 10$ \\
\hline
\end{tabular}


The performance of the system which takes up $20 \%$ of the final grade mainly aim to evaluate the accuracy and stability of the system. We give students an underlying score if the system only realize the basic function. For example, though the rational speed, direction and stop bit of the fly wheel can be measured, they depend on different programs. In other words, each program only implements one function. In this case, the system has a little practicability. Students will get a higher score if the system implements all functions depending on one program. In addition, the standardization of circuit connection is taken into account. As shown in Figure 3, the circuit connection in Figure 3(a) is more standardized than the one in Figure 3(b). Apparently, the stability of former is better than the latter.

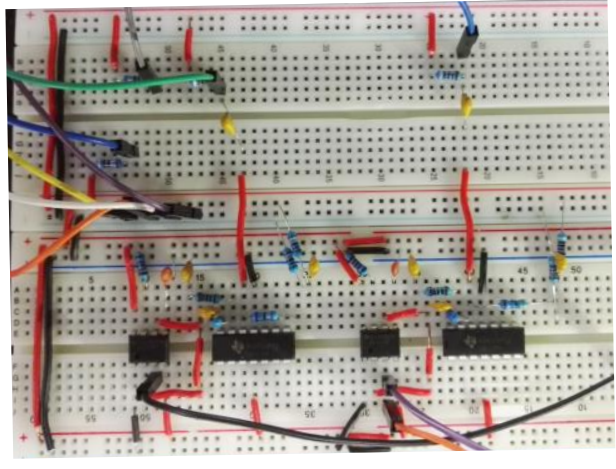

(a)

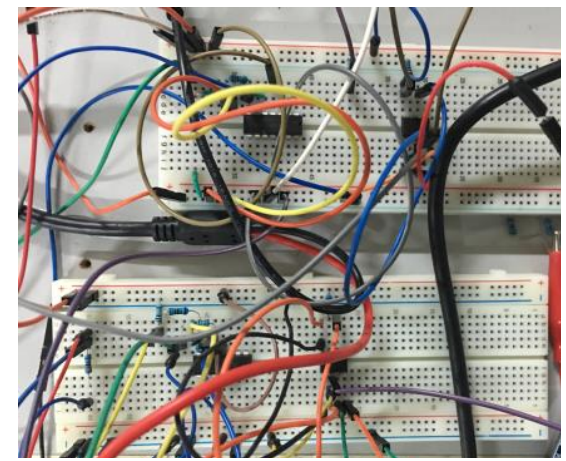

(b)

Figure 3. Two hardware circuits.

Each student is required to submit an experimental report. The report of the experiment takes up 10\%. In addition to describing the scheme, students are encouraged to summarize the problems and their solutions in the experiment. It can help them to accumulate engineering experience.

In order to improve students' expression ability, at the end of the experiment, each team need to give a 5 minutes presentation and other teams can ask questions and communicate with each other. This part accounts for $15 \%$ in the calculation of the final grade. The scoring rule is given in Table 3.

Table 3. The scoring rule of presentation.

\begin{tabular}{|c|c|}
\hline rule & score (total score is 15) \\
\hline language expression ability & $0 \sim 7$ \\
\hline the quality of PPT & $0 \sim 4$ \\
\hline communication skill & $0 \sim 4$ \\
\hline
\end{tabular}

\section{CONCLUSION}

Traditional experiment is attached to the theory teaching, and students only need to validate the content on the textbook with the detailed experimental instruction given by teachers. This kind of experiment results in the lack of autonomous learning motivation and creativity spirit of students. To address this issue, comprehensive experiments are set up in many colleges and universities. In this paper, a new teaching mode of comprehensive experiment is introduced while a photoelectric comprehensive experiment is given as an example. In the proposed teaching mode, the practicability in project and the comprehensiveness of knowledge is considered when an experiment is designed, and student-centered teaching method is adopt to take student as the subject in the classroom and encourage students to do the experiment independently. Besides, a scientific and reasonable experimental evaluation is proposed to test the students' mastery theory knowledge, practical ability, innovative ability and expression ability comprehensively. Practice over a period of five years shows that, the new experimental teaching mode can effectively cultivate students' comprehensive quality.

\section{REFERENCES}

[1] He, C., Lei, H., Wang, Q., and Gong, X., "The solutions to strengthening the construction of computer experimental teaching faculty, " Software Engineering Education for a Global E-Service Economy, papers,125132 (2014). 
[2] Lan, L., Liu. S., Zhou, J. H., and Peng, Z. M., Booth, N. and Smith, A. S., "Experimental teaching reforms of optical fiber communication based on general education," Proc. ETOP,104524Y1-104524Y6 (2017).

[3] Wang, D., Zhang, Y., Wang, X., Zhang, X., Zhang, T., and Sun, P., "Innovation ability and innovation spirit in photoelectric comprehensive experiment teaching", Proc. ETOP,104526F1-1045246F5 (2017).

[4] Qian, J., and Xu, P., "Measuring rotor speed and direction with quadrant photoelectric detectors", Optoelectronic engineering, 33(9), 67-70 (2006).

[5] Wang, W., Yan, X. W., Liu, W., Shi, J. H., Lei, B., Yao, T. F., and Hu, H. J., "Reform of experimental teaching based on quality cultivation", Proc. ETOP,104524M1-104524M4 (2017

[6] Li, X., and Gan, S., "The evaluation of undergraduate accounting experimental teaching", Proceedings of the 6th International Asia Conference on Industrial Engineering and Management Innovation, 1087-1092 (2016). 